\title{
Influence of Test Parameters on the Performance of Rapid Anti-Hepatitis C Virus Immunochromatographic Assay
}

\author{
Eun Jin $\mathrm{Choi}^{1 \oplus}$, Jae Han Kim${ }^{1 \oplus}$, and Mi-Soon $\mathrm{Han}^{2 \oplus}$ \\ ${ }^{1}$ Department of Laboratory Medicine, U2 Clinical Laboratories, Jangwon Medical Foundation, Seoul; 2Department of Laboratory \\ Medicine, Seoul Clinical Laboratories, Yongin, Korea
}

\section{Corresponding author:}

Mi-Soon Han

Department of Laboratory Medicine, Seoul Clinical Laboratories, Heungdeok IT Valley 25F, 13 Heungdeok 1-ro, Giheung-gu, Yongin 16954, Korea $\mathrm{Tel}+82-2-330-2210$

Fax +82-2-790-6509

E-mail misoon_han@scllab.co.kr

Received: July 30, 2020

Revised: October 23, 2020

Accepted: November 16, 2020

This is an Open Access article distributed under the terms of the Creative Commons Attribution Non-Commercial License (http://creativecommons.org/licenses/ by-nc/4.0) which permits unrestricted non-commercial use, distribution, and reproduction in any medium, provided the original work is properly cited.
Anti-hepatitis C virus (HCV) immunochromatographic assay (ICA) is commonly used as an early screening test for HCV infection. This study evaluated the influence of test parameters (technician, assay type, kit lot, temperature, and humidity) on the performance of anti-HCV ICA. The antiHCV results were collected in 2016-2018 by two ICA assays (Humasis HCV card [Humasis Co. Ltd., Korea] and Asan Easy Test HCV [Asan Pharmaceutical, Korea]) $(\mathrm{N}=55,919)$. Anti-HCV ICA-positive samples ( $\mathrm{N}=639)$ were retested using Architect anti-HCV (Abbott Laboratories, Germany). There were statistically significant differences in the retest signal to cutoff $(\mathrm{S} / \mathrm{CO}$ ) ratios of anti-HCV ICA-positive samples among the groups with different technicians and kit lots $(P<0.05)$. The positive rates of anti-HCV ICA were statistically significantly different according to indoor humidity levels $(P=0.0405)$. These findings suggest that there is a need for clinical laboratories to manage the performance of anti-HCV ICA with acceptable ranges of positive rates and utilize the $\mathrm{S} / \mathrm{CO}$ ratio to evaluate the influence of test parameters.

(Lab Med Qual Assur 2021;43:37-42)

Key Words Hepatitis C virus, Hepatitis C antibodies, Immunochromatographic assay, Performance, Evaluation
In approximately $30 \%$ of patients with hepatitis C virus (HCV), infection will spontaneously clear, but the remaining 70\% develop chronic infection [1]. Almost all patients infected with HCV generate antibodies against HCV (anti-HCV), and anti-HCV tests can detect $>90 \%$ of HCV infection at 3 months after exposure [2]. Screening using anti-HCV test is recommended for the clinical diagnosis and discrimination of asymptomatic patients [3]. For anti-HCV detection, the immunochromatographic assay (ICA) is still used at a substantial rate (up to $34.0 \%$ ) in clinical laboratories in South Korea [4]. For the ICA method, as well as the sensitivity of the test kit, the reading time and criteria, and the storage condition of the reagent have an important effect on the results, and the results vary greatly depending on the product [5]. The variability caused by technician readings can be large relative to the uniformity of the performance between manufacturing lots [6]. Temperature and humidity might have an effect on the evaporation of specimens and reagents and on lateral flow in the anti-HCV ICA test process. The aim of this study was to evaluate the influence of test parameters, such as the technician, kit product, lot number, and laboratory environment (temperature and humidity) on the performance of rapid anti-HCV ICA.

We collected each anti-HCV assay results using three 


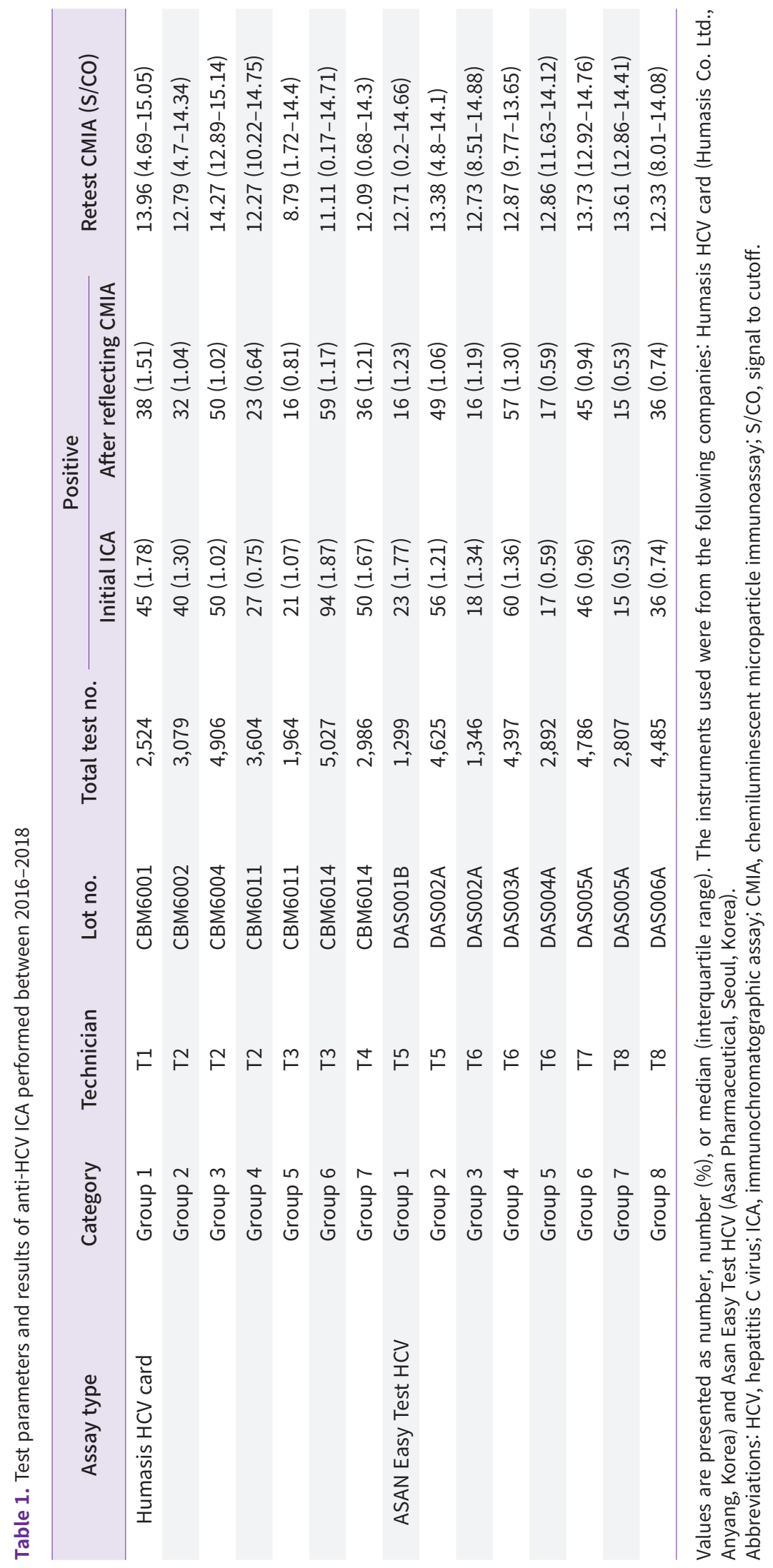


methods from 2016 through 2018 at a commercial centralized accredited laboratory in Seoul, South Korea: two ICAs (Humasis HCV card [Humasis Co. Ltd., Anyang, Korea] $[\mathrm{N}=24,489]$ and Asan Easy Test HCV [Asan Pharmaceutical, Seoul, Korea] [N=31,430]), and one chemiluminescent microparticle immunoassay (CMIA) of Architect anti-HCV (Abbott Laboratories, Wiesbaden, Germany) ( $N=60,632)$. The detection limit or cutoff were as follows: "not provided" for the Humasis HCV card, 2.0 signal to cutoff (S/CO) ratio for Asan Easy Test HCV, and 1.0 $\mathrm{S} / \mathrm{CO}$ ratio for CMIA. Comparisons between methods were performed after matching age and regional conditions for each data, that is, Humasis HCV card and Asan Easy Test HCV ( $N=21,386$ pairs), and between both ICAs and a CMIA ( $\mathrm{N}=40,665$ pairs). Anti-HCV ICA-positive samples ( $\mathrm{N}=639$ ) were retested by anti-HCV CMIA using the Architect i2000SR analyzer (Abbott Diagnostics, Abbott Park, IL, USA). The influence of test parameters on the performance of anti-HCV ICA was analyzed based on the S/CO ratios by retesting anti-HCV CMIA (total of 13 lots). Test parameters for anti-HCV ICA were included in 11 kit lot numbers of two assay types (Humasis HCV card, five numbers; Asan Easy Test HCV, six numbers), eight technicians, temperature $\left({ }^{\circ} \mathrm{C}\right)$, and humidity $(\%)$. This study was exempted from review by the institutional review board of the Jangwon Medical Foundation (IRB_2019017). Data were analyzed using the online GraphPad Prism software ver. 5.0 (GraphPad Software Inc., San Diego, CA, USA) and R ver. 4.0.2 software (R Foundation for Statistical Computing, Vienna, Austria). We compared using chi-square with Yates correction, independent t-test, and nonparametric multiple comparisons. Statistical significance was set at $P<0.05$.

The positive rates for the Humasis HCV card and the Asan Easy Test were not significantly different (1.09\% [95\% confidence interval $(\mathrm{Cl}), 0.68-1.61$ ] versus $0.94 \%$ [95\% Cl, 0.57-1.44]; $P=0.1118$ ). Note that the positive rates of anti-HCV ICA and CMIA assays showed a statistically significant difference $(1.25 \%$ [95\% Cl, 0.94-1.65] versus $2.83 \%$ [95\% Cl, 2.34-3.38]; $P<0.0001)$. The $\mathrm{S} / \mathrm{CO}$ ratios of anti-HCV ICA-positive samples ( $\mathrm{N}=639)$ showed statistically significant differences between the following groups: (1) Humasis HCV card lots between group 3 (technician $[T] 2$ and CMB6004) and group 6 (T3 \& CBM6014) $(P=0.0013)$, between group 3 and group 7 (T4 \& CBM6014) $(P=0.0075)$; (2) ASAN Easy Test HCV lots between group 4 (T6 \& DAS003A) and group 6 (T7 \& DAS005A) $(P=0.0252)$ (Table 1, Fig. 1). There were no cases showing statistically
A

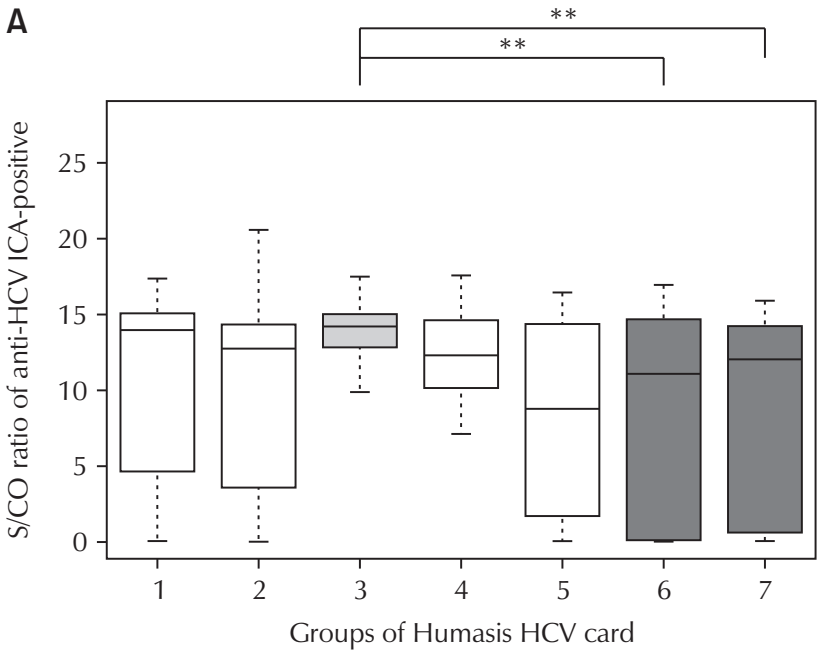

B

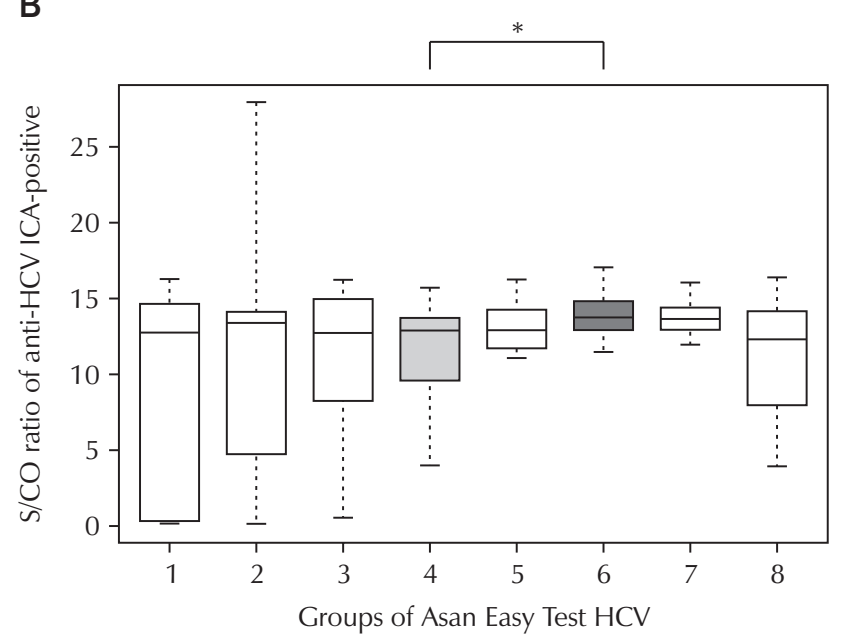

Fig. 1. Distribution of the S/CO ratio of anti-HCV ICA-positive samples, subgrouped according to technician and kit lot (shown in Table 1). (A) Humasis kit. (B) ASAN kit $\left({ }^{\star} P<0.05,{ }^{\star \star} P<0.01\right)$. Boxs represent the interquartile range and the line within box represents the median. The I bars represent the 2.5th and 97.5th percentiles. The instruments used were from the following companies: Humasis HCV card (Humasis Co. Ltd., Anyang, Korea) and Asan Easy Test HCV (Asan Pharmaceutical, Seoul, Korea). Abbreviations: S/CO, signal to cutoff; HCV, hepatitis C virus; ICA, immunochromatographic assay. 
significant differences between kit lots with the same technician or between technicians in the same kit lot. Thus, it is not clear whether the factor that caused the difference between groups was the technician or the kit lot. Of the 13 lot numbers of anti-HCV CMIA used to obtain the retest $\mathrm{S} / \mathrm{CO}$ ratio, statistically significant differences were found between the first and the other two lots ( $P=0.0223, P=0.0246)$. However, the first kit of anti-HCV CMIA was used with the Humasis HCV card, whereas the other two kits were used for ASAN Easy Test HCV.

The monthly average room temperature and humidity for the past 2 years ranged from $22^{\circ} \mathrm{C}$ to $25^{\circ} \mathrm{C}$ and $14 \%-65 \%$, respectively. There was no statistically significant difference between monthly temperature or humidity and the positive rate of the anti-HCV ICA test $(P=0.0689, P=0.8686)$. We divided daily humidity $(\mathrm{N}=524)$ into three groups $(<20 \%, 20 \%-55 \%$, and $>55 \%)$ using the recommended humidity range (20\%-55\%) in the laboratory area [7]. However, the group with the lowest humidity $(<20 \%)$ showed a statistically significant difference in the positive rate (\%) (mean \pm standard deviation [SD], 1.7 $\pm 1.1 ; \mathrm{N}=69$ ) compared with the sum of the other two groups (mean $\pm S D, 2.4 \pm 1.5 ; P=0.0405$; $\mathrm{N}=455$ ) (Fig. 2).

Several studies have reported that rapid anti-HCV assays showed acceptable performance and can be used as an alternative for commercially automated immunoassays [8,9]. In our study, the positive rate of antiHCV ICA was less than half that of anti-HCV CMIA. The positive region with a low $\mathrm{S} / \mathrm{CO}$ ratio was mainly detected only by the anti-HCV CMIA. These differences in analytical performance at low $\mathrm{S} / \mathrm{CO}$ ratios are presumably influenced by the false-positive rate of the anti-HCV CMIA assay and by the low sensitivity and false negative rate of anti-HCV ICA assay [10,11]. Choi et al. [10] reported the S/CO ratio (mean \pm SD) in an active HCV infection state was 12.96士 2.90 (versus past HCV infection: 5.29 \pm 4.53 ; false-positive anti-HCV: $1.81 \pm 0.89$ ). Seo et al. [12] suggested 10.9 as a cutoff value of the S/CO ratio of anti-HCV for predicting hepatitis C viremia. Referring to previous studies, a low $\mathrm{S} /$ $\mathrm{CO}$ ratio indicated false-positive anti-HCV or past, resolved infection, and was less likely to be due to a current infection.

For a qualitative test requiring visual reading, such as the type often called the "rapid test," there is no recognized reference material, and it is not feasible to obtain the response as a function of "concentration." Almost all accuracy and comparability evaluations for the rapid ICA assay are performed by determining only the agreement with an intended response to known positive and
A

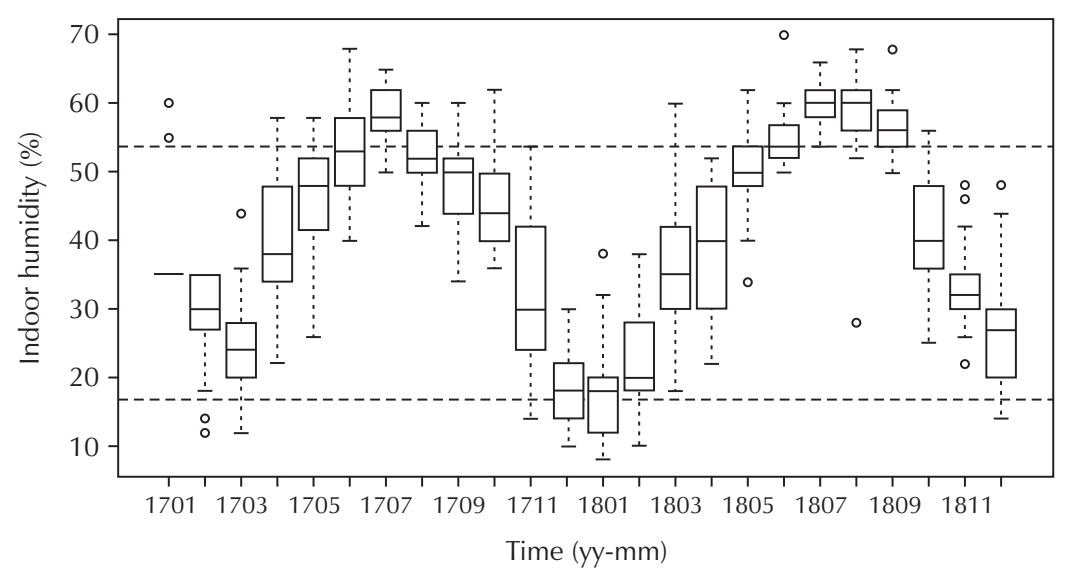

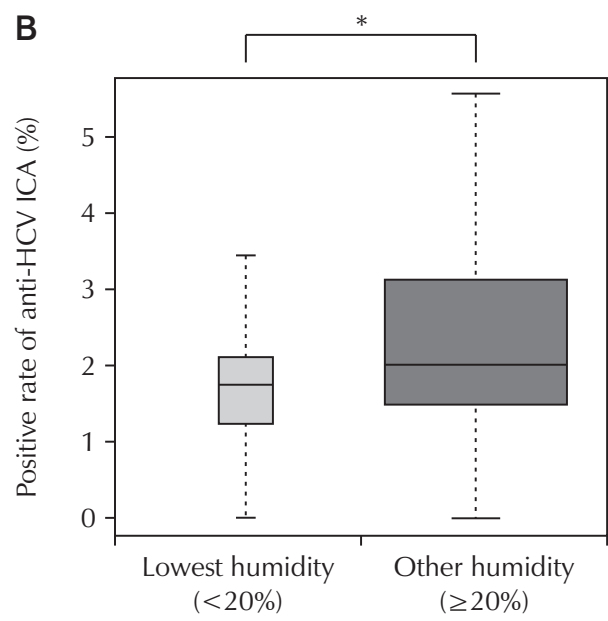

Fig. 2. Analysis of Indoor humidity (\%) in workplace performed the anti-HCV ICA tests from 2017 to 2018. (A) Monthly humidity. (B) Positive rate of the anti-HCV ICA in different humidity $\left({ }^{\star} P<0.05\right)$. Boxs represent the interquartile range and the line within box represents the median. The I bars represent the 2.5th and 97.5th percentiles. Black dashed lines represent the recommended range of the humidity [7]. The instruments used were from the following companies: Humasis HCV card (Humasis Co. Ltd., Anyang, Korea) and Asan Easy Test HCV (Asan Pharmaceutical, Seoul, Korea). Abbreviations: HCV, hepatitis C virus; ICA, immunochromatographic assay. 
negative samples $[13,14]$. Using only the qualitative result of the ICA assay is not sufficient to properly evaluate and quantify the performance variability of the rapid ICA assay depending on the technician, kit lot, and laboratory climate factors. Our study showed that there were statistically significant differences in the performance of anti-HCV ICA based on the technician and/or kit lot number for a defined clinical application (Fig. 1). Even for a rapid qualitative test, we recommend consistent performance assurance for the anti-HCV ICA assay, using the $\mathrm{S} / \mathrm{CO}$ ratio of the anti-HCV CMIA assay.

Clinical laboratories usually apply tolerances to room temperature and humidity to ensure sample and reagent storage stability. However, little is known about the effects of these climatic factors on the performance of ICA assays. The temperature of the work area for anti-HCV ICA was well controlled within the range of "room temperature," which is generally defined as $20^{\circ} \mathrm{C}-25^{\circ} \mathrm{C}$. However, the indoor humidity for the same area was not controlled within the acceptable range in the CLSI guidelines for two consecutive winters (2017-2018). Because the positive rate of the anti-HCV ICA in the lowest humidity group was significantly low, the laboratory should pay more attention to humidity control in winter.
Our study has some limitations. A comparable assay (anti-HCV CMIA) was performed against only test assay (anti-HCV ICA)-positive samples, and the competence differences among the technicians may depend on the condition of each laboratory. Finally, it is debatable whether anti-HCV ICA performance should meet the performance level of anti-HCV CMIA. In conclusion, antiHCV ICA showed a lower positive rate than anti-HCV CMIA. When anti-HCV ICA performance was evaluated using the $\mathrm{S} / \mathrm{CO}$ ratio, there were statistically significant differences in terms of test parameters, such as technician, assay lot, and indoor humidity. These findings suggest that there is a need for clinical laboratories to manage the performance of anti-HCV ICA with acceptable ranges of positive rates and utilize the S/CO ratio to evaluate the influence of test parameters. Finally, we recommend paying more attention to winter humidity control in laboratory areas.

\section{ORCID}

Eun Jin Choi https://orcid.org/0000-0003-0775-8909

Jae Han Kim https://orcid.org/0000-0002-5922-2471

Mi-Soon Han https://orcid.org/0000-0003-2527-2494

\section{REFERENCES}

1. World Health Organization. WHO fact sheet Hepatitis C. https://www.who.int/en/news-room/fact-sheets/detail/ hepatitis-c (Accessed July 30, 2020).

2. Chen SL, Morgan TR. The natural history of hepatitis C virus (HCV) infection. Int J Med Sci 2006;3:47-52.

3. Centers for Disease Control and Prevention (CDC). Testing for HCV infection: an update of guidance for clinicians and laboratorians. MMWR Morb Mortal Wkly Rep 2013;62:362-5.

4. Cho EJ, Choi AR, Ryu JH, Yun SJ, Lee W, Chun S, et al. Report on the external quality assessment scheme of hepatitis viral markers in Korea, (2016-2017). J Lab Med Qual Assur 2018;40:51-69.

5. Bienek DR, Charlton DG. The effect of simulated field storage conditions on the accuracy of rapid user-friendly blood pathogen detection kits. Mil Med 2012;177:583-8.

6. Berg B, Hellsing K, Jagenburg R, Kallner A. Guidelines for evaluation of reagent strips: exemplified by analysis of urine albumin and glucose concentration using visually read reagent strips. International Federation of Clinical Chemistry, Scientific Division. Scand J Clin Lab Invest 1989;49:689-99.

7. Clinical and Laboratory Standards Institute. Laboratory design: QMS04-ED3. 3rd ed. Wayne (PA): Clinical and Laboratory Standards Institute, 2016.

8. Al-Tahish G, El-Barrawy MA, Hashish MH, Heddaya Z. Effectiveness of three types of rapid tests for the detection of 
hepatitis C virus antibodies among blood donors in Alexandria, Egypt. J Virol Methods 2013;189:370-4.

9. Tang W, Chen W, Amini A, Boeras D, Falconer J, Kelly H, et al. Diagnostic accuracy of tests to detect Hepatitis C antibody: a meta-analysis and review of the literature. BMC Infect Dis 2017;17(Suppl 1):695.

10. Choi MS, Lee K, Hong YJ, Song EY, Kim DS, Song J. The role of the signal-to-cutoff ratio in automated antiHCV chemiluminescent immunoassays by referring to the nucleic acid amplification test and the recombinant immunoblot assay. Ann Lab Med 2018;38:466-72.

11. Kim S, Kim JH, Yoon S, Park YH, Kim HS. Clinical performance evaluation of four automated chemiluminescence immunoassays for hepatitis C virus antibody detection. J Clin Microbiol 2008;46:3919-23.

12. Seo YS, Jung ES, Kim JH, Jung YK, Kim JH, An H, et al. Significance of anti-HCV signal-to-cutoff ratio in predicting hepatitis C viremia. Korean J Intern Med 2009;24:302-8.

13. Clinical and Laboratory Standards Institute. User evaluation of between-reagent lot variation; approved guideline: EP26-A. Wayne (PA): Clinical and Laboratory Standards Institute, 2013.

14. Clinical and Laboratory Standards Institute. User protocol for evaluation of qualitative test performance; approved guideline-second edition: EP12-A2. Wayne (PA): Clinical and Laboratory Standards Institute, 2008. 